La voz de paciente: trazos para el desarrollo futuro

\title{
The patient's voice: outlines for future development
}

doi.org/10.23938/ASSN.0385

\author{
I. Rodrigo Rincón, N. Arraiza Saldise
}

El concepto y los instrumentos para medir lo que se denomina experiencia del paciente están cobrando cada vez mayor importancia. Aunque a menudo la realización de encuestas de satisfacción y el análisis de la experiencia de pacientes se consideran sinónimos, merece la pena analizar las diferencias y similitudes entre los dos conceptos.

Las encuestas de satisfacción de pacientes empezaron a utilizarse hace unos treinta años como una fuente de información útil para identificar áreas de mejora en las organizaciones sanitarias. Donabedian, en 1990, ya incorporó la percepción de los pacientes en el marco de la evaluación de la calidad ${ }^{1}$.

La evaluación de la atención sanitaria desde la perspectiva de los pacientes les otorga voz y permite una orientación más adecuada de las organizaciones hacia ellos, lo que a su vez puede contribuir a una mayor satisfacción de los pacientes.

Sin embargo, conceptualizar la satisfacción es difícil debido a que es una medida subjetiva y no necesariamente ligada a una realidad externa. Dos individuos tratados de forma idéntica pueden percibir los servicios sanitarios de forma diferente. De hecho, la comunidad científica no ha llegado a un acuerdo sobre la definición de satisfacción ${ }^{2,3}$.

Según el enfoque holístico, podría decirse que las puntaciones de los cuestionarios de satisfacción de los pacientes reflejan preferencias, expectativas, valores y deseos de los pacientes, y no solamente la calidad del servicio recibido ${ }^{4}$.

Francia hizo obligatorio en 1996 que los hospitales midiesen la satisfacción de sus pacientes ${ }^{5}$. En 2002 el Departamento de Salud de Inglaterra lanza un programa anual nacional para medir la satisfacción de los pacientes. De la misma manera, desde 2005 Alemania incorporó la medida de la satisfacción de los pacientes como uno de los elementos clave en la gestión de la calidad ${ }^{6}$.

Servicio de Apoyo a la Gestión Clínica y Continuidad Asistencial. Complejo Hospitalario de Navarra. Pamplona.

\section{Agradecimientos:}

A Guillermo Ezpeleta por sus valiosos comentarios.

\section{Correspondencia:}

Isabel Rodrigo Rincón

Servicio de Apoyo a la Gestión Clínica y

Continuidad Asistencial.

Complejo Hospitalario de Navarra.

E-mail: mi.rodrigo.rincon@cfnavarra.es 
En España, aunque su uso está muy extendido, no hay un instrumento común para medir la satisfacción de los pacientes en las organizaciones sanitarias. El instrumento con más recorrido que permite comparar la percepción de los ciudadanos con los servicios sanitarios públicos entre comunidades autónomas es el barómetro sanitario. Se trata de un estudio de opinión que realiza el Ministerio de Sanidad, Servicios Sociales e Igualdad, en colaboración con el Centro de Investigaciones Sociológicas de forma anual desde $1993^{7}$.

A pesar de su amplia utilización, las encuestas de satisfacción son criticadas como herramientas de evaluación de la calidad de los servicios, especialmente por dos aspectos. El primero tiene que ver con que la evaluación subjetiva de la calidad de la atención recibida podría verse afectada por el desconocimiento de los pacientes sobre aspectos importantes del cuidado ${ }^{8}$. De hecho, es posible que con el fin de intentar obtener mejor puntuación en las encuestas de satisfacción de pacientes, las organizaciones sanitarias presten una atención sanitaria de menor calidad $^{9,10}$. Así lo ponen de manifiesto algunos estudios que han demostrado una asociación entre la satisfacción de los pacientes con las organizaciones y un incremento en la prescripción de antibióticos ${ }^{10}$.

En este mismo sentido, el estudio reciente publicado en este mismo número sobre satisfacción del paciente en el Sistema Nacional de Salud español ${ }^{11}$ concluye que a nivel de comunidad autónoma existe una asociación de carácter positivo entre la satisfacción percibida por el usuario y el número de médicos especialistas, el número de ingresos hospitalarios y la mortalidad intrahospitalaria. Si bien la mera existencia de una asociación no implica causalidad, este estudio refleja la dificultad que entraña el análisis de la satisfacción. Este hecho cobra relevancia en el análisis de la satisfacción del paciente y su asociación con la mortalidad intrahospitalaria puesto que, como es posible apreciar, la medida de la satisfacción conlleva el análisis no solo el análisis de la calidad de la asistencia recibida sino otros aspectos vitales del paciente ya mencionados con anterioridad. Por ejemplo, los pacientes pueden sentirse más satisfechos cuantos más ingresos y especialistas hay, pero es posible que actualmente tanto la potenciación de la atención hacia el primer nivel como otras modalidades asistenciales alternativas a la hospitalización puedan tener un mayor efecto en su salud tanto a corto como a largo plazo. El mismo trabajo ${ }^{11}$ resalta la asociación negativa entre algunos efectos adversos, como la infección hospitalaria, la estancia hospitalaria y el gasto farmacéutico, y la satisfacción de los pacientes. Todo ello sugiere la importancia de hacer lo correcto, en el sentido de aplicar las prácticas seguras y de evitar lo innecesario debido a la posibilidad de aumentar los efectos adversos, además de generar un gasto innecesario ${ }^{12-14}$.

El segundo aspecto criticado sobre las encuestas de satisfacción de pacientes tiene que ver con que las respuestas tabuladas de los cuestionarios, por ejemplo a través de un valor numérico, no siempre consiguen captar la experiencia subjetiva del paciente ${ }^{10}$. Sin embargo, mientras que los factores subjetivos y psicológicos pueden tener influencia en los resultados individuales de satisfacción, se ha demostrado la fiabilidad del análisis cuando se realiza de forma agregada, a nivel de servicios, unidades o centros ${ }^{15-17}$. 
A pesar del interés en la medición de la percepción o satisfacción del paciente con un determinado sistema sanitario, aún no existe un método estándar o instrumento definitivo para su evaluación. Numerosos autores han evidenciado la carencia de instrumentos válidos y reproducibles, pero a pesar de ello, los avances en la metodología tanto de recogida de los datos como en el análisis de los mismos han sido discretos ${ }^{18-20}$. En este sentido, la mejora en los aspectos metodológicos depende del desarrollo de nuevos cuestionarios y validación cuidadosa de los existentes teniendo en cuenta las condiciones de aplicación de los mismos.

El análisis de la experiencia de los pacientes, a pesar de ser un aspecto evaluado por muchas organizaciones no presenta una definición estándar, siendo numerosas las definiciones y dimensiones que se engloban bajo ese constructo ${ }^{21}$. La diferente conceptualización viene determinada a menudo por la diferencia de los contextos sanitarios donde se implanta, así como las herramientas que se utilizan para su medición y análisis.

El Instituto Beryl define experiencia de pacientes como la integración de las interacciones culturales y percepciones del paciente a lo largo de todo el continuo de cuidados. A esa definición habría que añadir los siguientes elementos: la implicación activa de pacientes y familiares, la obligatoriedad de centrarse en las necesidades de las personas y el reconocimiento de una experiencia integral ${ }^{21}$.

La experiencia de los pacientes mide tanto las percepciones subjetivas (control del dolor) como objetivas (tiempo de espera hasta la cita) además de las de relación y comunicación, y en general aspectos que indican que los cuidados se centran en el paciente ${ }^{22}$. No obstante, se precisa seguir avanzando en el marco conceptual que permita identificar los principios clave para optimizar la experiencia de los pacientes en su recorrido por las organizaciones sanitarias ${ }^{21}$.

Por otro lado, se puede considerar que la satisfacción del paciente es un subconjunto dentro un marco global mayor que es la experiencia de los pacientes. Parte de la experiencia del paciente es su satisfacción, que vendría a ser un elemento más bien pasivo, abarca unos horizontes más amplios y requiere una mayor implicación e interacción tanto de pacientes como de profesionales.

Uno de los instrumentos más utilizados para medir la experiencia de los pacientes son los cuestionarios CAHPS (Consumer Assessment of Healthcare Providers and System), que incluyen aspectos que los usuarios consideran importantes, como las habilidades de comunicación de los profesionales, la accesibilidad a los servicios sanitarios, la coordinación entre profesionales, etc. ${ }^{23}$. Son una herramienta estandarizada y por lo tanto facilitan la comparación directa entre proveedores sanitarios. Asimismo presentan la ventaja de que los resultados obtenidos son de dominio público, lo que permite realizar un feed-back a la población y de esa manera considerarla o incluso empoderarla. Además, se están utilizando como bonos de reembolso para las organizaciones que tratan a pacientes de Medicare.

Dado que la experiencia del paciente es una vivencia rica que comprende múltiples aspectos y que, además, el instrumento a elegir para su medición dependerá no solo de sus propiedades sino también del sistema de atención de salud, el propósito del estudio y el contexto en 
el cual se aplica, la AHRQ (Agency for Health Research and Quality) está investigando cómo recoger esta experiencia de pacientes de forma más cualitativa a través de cuestionarios con preguntas abiertas. El objetivo es recoger la narrativa de los pacientes como complemento a la utilización de instrumentos más estandarizados ${ }^{24}$. Sin duda, estos cuestionarios enriquecen la información que proporcionan pero presentan un nuevo reto: la necesidad de herramientas para su análisis. Se requieren herramientas de procesamiento de lenguajes naturales (Natural Language Processing, $N L P$ ), que además de dar coherencia a los resultados, los haga técnicamente posibles además de eficientes.

Otros cuestionarios frecuentemente utilizados para medir la experiencia de los pacientes son The Picker Patient Experience Questionnai$r e^{25}$, el Patient Experience Questionnaire ${ }^{26}$, y el cuestionario IEXPAC ${ }^{27}$ para pacientes con enfermedades crónicas.

Por otra parte, las herramientas que describen y analizan la experiencia de los pacientes no se reducen a los cuestionarios sino que comprenden otros instrumentos como son mapas de experiencias, $E x$ perience Based Codesign (EBCD), en los que se graban historias y experiencias reales de pacientes, grupos de discusión, etc.

Estas herramientas o enfoques se categorizan de acuerdo con la profundidad de la información que proporcionan y la medida en que la información recopilada por los mismos puede ser generalizable a una población más amplia. En la selección del enfoque o la herramienta apropiada, es conveniente ponderar la importancia de la profundidad de la información versus la generalización a una población determinada, siendo necesaria la mayor parte de las veces una combinación de técnicas con objeto de satisfacer ambos enfoques.

Ningún método de recogida de información de los pacientes es perfecto, pero la combinación de métodos cualitativos y cuantitativos reduce de forma considerable los déficits potenciales de cualquiera de ellos $^{28}$.

\section{Cauces alternativos: redes sociales}

Los ciudadanos cada vez utilizan más cauces alternativos para conocer la calidad que prestan los profesionales sanitarios. Un estudio realizado por Lagu y col${ }^{8,29}$ indica que los sitios web (websites) son una nueva manera de obtener información sobre la calidad de los profesionales y que los pacientes pueden utilizarlas para tomar decisiones, como por ejemplo, para elegir médico.

Los sitios web que evalúan a los centros y a los profesionales sanitarios son un elemento más de la Web Social o Web 2.0, una opción adicional para que los pacientes expresen su grado de satisfacción por la atención recibida ${ }^{29}$. Estos sitios web ya han llegado a nuestro país y tienen una estructura similar a los que utilizan los turistas para evaluar hoteles, restaurantes, etc., con la ventaja de que los resultados pueden resultar más sencillos de interpretar que los proporcionados por las instituciones sanitarias, a menudo más sofisticados. Además, permiten recoger percepciones de los usuarios en texto libre, con la riqueza de matices que ello genera. En su mayoría, son sitios web genéricos ya que abarcan distintos sectores, pero pronto podrán aparecer sitios más 
específicos para el sector salud. A estos sitios web específicos se les podría bautizar como sanidadadvisor.

Si las organizaciones sanitarias no canalizan las herramientas para que los pacientes puedan dar su opinión de una manera sencilla, los ciudadanos se autoorganizan para compartir la información. A veces confluyen los canales institucionales con los autogenerados. No obstante, sería útil tener en cuenta y analizar la información recogida en los sanidadadvisor para ampliar el abanico de información sobre satisfacción y experiencia de los pacientes.

\section{Cuestionarios interactivos}

Algunas organizaciones utilizan cuestionarios interactivos con algoritmos ${ }^{30}$ para implicar a los usuarios en la mejora de la calidad de los servicios sanitarios. A los usuarios se les presenta un abanico de áreas (comunicación con profesionales, aparcamiento, etc.) y se les pregunta cuáles pueden ser mejoradas. De las áreas que elige como "mejorables”, el sistema lanza un conjunto de preguntas más específicas. De esta manera, la organización consigue un análisis en profundidad sobre las áreas que más interesan a los pacientes sin saturarle a preguntas. Este sistema tiene como desventaja que no permite realizar análisis comparativos entre servicios y unidades de todos los ítems aunque sí de las grandes áreas que son comunes en los cuestionarios para todos los pacientes.

En la propuesta de Hall y col $^{30}$, además de incorporar un algoritmo en el cuestionario on-line, dan protagonismo a los usuarios en el análisis y propuestas de mejora. Para ello, han creado un comité denominado Consumer Action Group con al menos dos personas representando a los usuarios. Estas personas son seleccionadas mediante un proceso competitivo y son formadas en análisis, monitorización e interpretación de los resultados, en la priorización para la toma de decisiones, en habilidades de comunicación, resolución de conflictos, etc.

La elección de usuarios mediante concurrencia competitiva, a quienes a menudo se les remunera por su participación, no ha tenido eco en nuestro país. Sin embargo, puede ser una iniciativa interesante, no solamente para este tipo de comités sino para favorecer un diseño y análisis de la atención más enfocada a las necesidades de los pacientes.

\section{Cuestionarios para medir el proceso integral}

Si la experiencia del paciente es integral y atraviesa los distintos centros y servicios, se necesitan instrumentos que midan el recorrido del paciente a lo largo de un proceso clínico. Ese es el objetivo de cuestionarios como CAHPS Cancer Care Survey ${ }^{23,}$ o National Cancer Patient Experience Survey ${ }^{31}$.

En la línea de lo ya comentado en este artículo, el análisis del texto libre junto con el de los datos tabulados aporta información muy útil a las organizaciones sobre las percepciones y experiencias de los pacientes en el recorrido del paciente que tiene un proceso clínico concreto $^{32}$. 


\section{Mensajes clave}

De acuerdo con lo expuesto, estos son los caminos que deberían emprender las organizaciones sanitarias para hacer efectiva la medición de la voz del paciente. La enumeración no indica un orden de precedencia ni implica que haya que llevar a cabo una acción para emprender la siguiente; idealmente, las organizaciones deberían establecer sus propias prioridades y emprender varias de manera simultánea.

1. Se precisan instrumentos que midan la satisfacción y la experiencia de los pacientes tanto de una forma más cuantitativa como cualitativa. Estos instrumentos tienen que estar validados y demostrar su utilidad para la mejora de las organizaciones sanitarias.

2. Las nuevas tecnologías nos permiten trabajar con cuestionarios que contengan un abanico de preguntas inmenso, pero el paciente solo tendrá que contestar a unas pocas en función de los resultados que vaya proporcionando a las preguntas previas. De esta manera las organizaciones van a poder tener información detallada de la percepción de los pacientes sobre diferentes aspectos, sin agotar la paciencia y el entusiasmo de los mismos.

3. Los resultados de la percepción de los usuarios se pueden disponer on-line porque quioscos, tabletas y teléfonos inteligentes permiten recoger la información y analizarla en el momento. Las habilidades digitales de la población va a poder muy pronto suprimir formas de administración y análisis más arcaicos como la recogida en papel.

4. La inclusión de nuevas tecnologías en la evaluación de la experiencia del paciente permitiría no solo la automatización de la recogida de datos y el análisis de los mismos, sino que además sería posible que el encuestado recibiese un feed-back instantáneo tras la finalización de la encuesta y la comparación de su percepción para cada uno de los ítems considerados con la global de la muestra encuestada.

5. La paulatina incorporación en las organizaciones sanitarias de herramientas que permitan el análisis del lenguaje natural posibilitaría una visión más rica sobre la experiencia del paciente en su conjunto permitiendo incorporar aspectos cualitativos que difícilmente serían analizables mediante el uso exclusivo de técnicas cuantitativas.

6. Las organizaciones necesitan utilizar tanto métodos cuantitativos como cualitativos (grupos focales, mapas de experiencia, etc.) que permitan entender el porqué de las conductas o de las no-conformidades, y que además nutran con preguntas a los cuestionarios para tener información de lo que al paciente le resulta relevante. De esta manera resultará más sencillo implicar a los pacientes en su salud y mejorar sus resultados.

7. Las organizaciones sanitarias tienen que ser proactivas para recoger y analizar las opiniones que los usuarios emiten a través de canales diferentes a los oficiales.

8. Se requiere un mayor desarrollo e implantación de instrumentos específicos que recojan la percepción de los pacientes de un proceso de enfermedad a lo largo de los diferentes profesionales y centros sanitarios. 
9. Un mayor protagonismo de los usuarios en la interpretación de los resultados y priorización de las acciones de mejora es otro de los elementos claves a tener en cuenta cuando se pretende implicar a los pacientes en la evaluación de los sistemas sanitarios.

Muchas de las herramientas y enfoques expuestos en este artículo ya forman parte de nuestro arsenal para medir y evaluar la percepción de los pacientes. En lo referente a mejorar la medición de la voz del paciente, el futuro ha llegado.

\section{BIBLIOGRAFÍA}

1. Donabedian A. Evaluating the quality of medical care. Milbank Mem Fund Q 1966; 44: 166-206.

2. Williams B, Coyle J, Healy D. The meaning of patient satisfaction: an explanation of high reported levels. Soc Sci Med 1998; 47: 1351-1359.

3. FiтZPAтRICK R, Hopkins A. Problems in the conceptual framework of patient satisfaction research: an empirical exploration. Sociol Health Illn 1983; 5: 297-311.

4. Crow R, Gage H, Hampson S, Hart J, Kimber A, Storey L et al. The measurement of satisfaction with healthcare: implications for practice from a systematic review of the literature. Health Technol Assess 2002; 6: 1-244.

5. Boyer L, Francois P, Doutre E, Weil G, Labarere J. Perception and use of the results of patient satisfaction surveys by care providers in a french teaching hospital. Int J Qual Health Care 2006; 18: 359-364.

6. SCHOENFelder T, KLEWER J, Kugler J. Determinants of patient satisfaction: a study among 39 hospitals in an in-patient setting in Germany. Int J Qual Health Care 2011; 23: 503-509.

7. Ministerio de Sanidad, Servicios Sociales e Igualdad. Secretaría General de Sanidad y Consumo. Barómetro Sanitario 2016. http://www.msssi.gob.es/estadEstudios/estadisticas/BarometroSanitario/home_BS.htm.

8. TanniRu M, Khuntia J. Dimensions of Patient Experience and Overall Satisfaction in Emergency Departments. J Patient Exp 2017; 4: 95-100.

9. Fenton JJ, Jerant AF, Bertakis KD, Franks P. The cost of satisfaction: a national study of patient satisfaction, health care utilization, expenditures, and mortality. Arch Intern Med 2012; 172: 405-411.

10. Trzeciak S, Gaughan JP, Bosire J, Mazzarelli AJ. Association between medicare summary star ratings for patient experience and clinical outcomes in US hospitals. J Patient Exp 2016; 3: 6-9.

11. Valls Martínez MC, Abad Segura E. Satisfacción del paciente en el Sistema Nacional de Salud español. An Sist Sanit Navar 2018; 41: 309-320.

12. Mira JJ, Carrillo I, Pérez-Pérez P, Olivera G, Silvestre C, Nebot C et al. Grado de conocimiento de la campaña Compromiso por la Calidad y de las recomendaciones no hacer entre médicos de familia, pediatras y enfermería de Atención Primaria. An Sist Sanit Navar 2018; 41: 47-55.

13. Mira JJ, Carrillo I, Sobrina G. Medicina defensiva en Atención Primaria. An Sist Sanit Navar 2018; 41: 273-275.

14. García Mochon L, Olry de Labry Lima A, Bermudez Tamayo C. Priorización de actividades clínicas no recomendadas en Atención Primaria. An Sist Sanit Navar 2017; 40: 401-412.

15. JANSSON JA, EKLUND M. Stability of perceived ward atmosphere over time, diagnosis and gender for patients with psychosis. Nord J Psychiatry 2002; 56: 407-412.

16. RodRIGo-Rincón I. Influencia de las unidades clínicas y de las especialidades médicas en la satisfacción de los pacientes. Pamplona: Universidad Pública de Navarra; 2010. 
17. Rodrigo Rincón I, ViÑES JJ, GUILLÉN-Grima F. Quality of the information supplied to patients by outpatient clinical units through multilevel model analysis. An Sist Sanit Navar 2009; 32: 183-197.

18. Rodrigo-Rincón I, Reyes-Pérez M, Martínez-Lozano ME. Personalizing the reference level: gold standard to evaluate the quality of service perceived. Rev Esp Med Nucl Imagen Mol 2014; 33: 65-71.

19. Rodrigo-Rincon I, Goñ-Girones E, Serra-Arbeloa P, Martinez-Lozano ME, ReyesPÉREz M. Discrepancies on quality perceived by the patients versus professionals on the quality of a nuclear medicine department. Rev Esp Med Nucl Imagen Mol 2015; 34: 102-106.

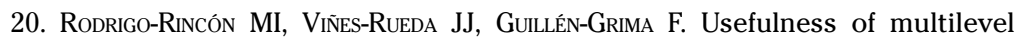
analysis of satisfaction questionnaires on clinical units and medical specialties. Rev Calid Asist 2012; 27: 212-225.

21. Wolf JA, Niederhauser V, Marshburn D, LaVela SL. Defining patient experience. Patient Experience Journal 2014; 1: 7-19. https://pxjournal.org/cgi/viewcontent.cgi?article $=1004 \&$ context=journal.

22. Anhang Price R, Elliott Mn, Zaslavsky AM, Hays RD, Lehrman WG, Rybowski L et al. Examining the role of patient experience surveys in measuring health care quality. Med Care Res Rev 2014; 71: 522-554.

23. US Department of Health and Human Services. Consumer Assessment of Healthcare Providers and Systems (CAHPS) surveys and guidance. Rockville: Agency. for Healthcare Research and Quality; 2018. http://www.ahrq.gov/ cahps/surveys-guidance/index.html.

24. Martino SC, Shaller D, Schlesinger M, Parker AM, Rybowski L, Grob R et al. CAHPS and comments: how closed-ended survey questions and narrative accounts interact in the assessment of patient experience. J Patient Exp 2017; 4: $37-45$.

25. Bertran MJ, Viñarás M, Salamero M, Garcia F, Graham C, McCulloch A et al. Spanish and catalan translation, cultural adaptation and validation of the Picker Patient Experience Questionnaire-15. J Healthc Qual Res 2018; 33: 10-17.

26. Pettersen KI, Veenstra M, Guldvog B, Kolstad A. The patient experiences questionnaire: development, validity and reliability. Int J Qual Health Care 2004; 16: 453-463.

27. Mira JJ, Nuño-Solinís R, Guilabert-Mora M, Solas-Gaspar O, Fernández-Cano P, GonzÁlez-Mestre MA et al. Development and validation of an instrument for assessing patient experience of chronic illness care. Int J Integr Care 2016; 16: 13 .

28. LeEs C. Measuring the patient experience. Nurse Res 2011; 19: 25-28.

29. Lagu T, Hannon NS, Rothberg MB, Lindenauer PK. Patients' evaluations of health care providers in the era of social networking: an analysis of physician-rating websites. J Gen Intern Med 2010; 25: 942-946.

30. Hall AE, Bryant J, Sanson-Fisher RW, Fradgley EA, Proietto AM, Roos I. Consumer input into health care: time for a new active and comprehensive model of consumer involvement. Health Expect 2018; 21: 707-713.

31. Bone A, McGrath-Lone L, Day S, Ward H. Inequalities in the care experiences of patients with cancer: analysis of data from the National Cancer Patient Experience Survey 2011-2012. BMJ Open 2014; 4: e004567.

32. Wiseman T, Lucas G, Sangha A, Randolph A, Stapleton S, Pattison N et al. Insights into the experiences of patients with cancer in London: framework analysis of free-text data from the National Cancer Patient Experience Survey 2012/2013 from the two London Integrated Cancer Systems. BMJ Open 2015; 5: e007792. 